\title{
Unexpected Anaphylaxis After Completing a Desensitization Protocol to Oxaliplatin: Successful Adjuvant Use of Omalizumab
}

Prieto-García A, Noguerado B, Rojas P, Torrado I, RodríguezFernández A, Tornero P

Servicio de Alergia, Hospital General Universitario Gregorio Marañón, Instituto de Investigación Sanitaria Gregorio Marañón, Madrid, Spain

J Investig Allergol Clin Immunol 2019; Vol. 29(1): 53-55 doi: $10.18176 /$ jiaci.0326

Key words: Anaphylaxis. Drug allergy. Drug desensitization. Oxaliplatin Omalizumab.

Palabras clave: Anafilaxia. Alergia a fármacos. Desensibilización a fármacos. Oxaliplatino. Omalizumab.

Omalizumab is a recombinant humanized anti-IgE monoclonal antibody. It binds to free serum IgE, thus downregulating expression of FceRI on the surface of mast cells and basophils [1]. It also dissociates prebound IgE from mast cells and basophils, with a reduction in cell-signaling events [2]. Omalizumab is approved for the treatment of severe allergic asthma and chronic urticaria. However, it has been used as off-label therapy in other conditions, such as rhinitis, nasal polyposis, atopic dermatitis, and mastocytosis. It has also been used as an adjuvant to increase tolerance and safety in specific oral tolerance induction for food allergy and in venom immunotherapy [3].

There are very few reports on the adjuvant use of omalizumab for drug desensitization [4-8]. We present a case of anaphylaxis to oxaliplatin in which omalizumab was needed to achieve successful desensitization.

A 61-year-old woman was diagnosed with stage $4 B R A F$ mutant colon adenocarcinoma, peritoneal carcinomatosis, adrenal nodules, and liver metastases. She was initially treated with FOLFOX (folinic acid-fluorouracil-oxaliplatin)bevacizumab. During the seventh cycle, shortly after starting the oxaliplatin infusion, she experienced palmar pruritus, erythematous pruritic rash on the face and thorax, pharyngeal pruritus, dyspnea, nausea, abdominal pain, diarrhea, and dizziness. Her blood pressure was $110 / 87 \mathrm{mmHg}$, heart rate $80 \mathrm{bpm}$, and oxygen saturation $98 \%$. The symptoms resolved after treatment with intravenous dexchlorpheniramine, hydrocortisone, ranitidine, and metoclopramide. The remaining chemotherapy was subsequently administered and well tolerated.

She was referred to our clinic for an allergological work-up. Skin tests with oxaliplatin were performed as recommended [9]. The result of the prick test $(5 \mathrm{mg} / \mathrm{mL})$ was negative. The result of the intradermal test $(0.5$ and $5 \mathrm{mg} / \mathrm{mL})$ was positive at $5 \mathrm{mg} / \mathrm{mL}(10 \times 8 \mathrm{~mm}$, wheal surrounded by erythema; histamine, $20 \times 17 \mathrm{~mm}$ ). 
Given the lack of response at the time, treatment was changed to FOLFIRI (folinic acid-fluorouracil-irinotecan)aflibercept (6 cycles) followed by FOLFIRI-cetuximab (3 cycles). The tumor was seen to progress. A fourth line of treatment with FOLFIRINOX (folinic acid-fluorouracilirinotecan-oxaliplatin)-bevacizumab was then proposed, and desensitization to oxaliplatin was scheduled.

The first desensitization was performed 10 months after the initial reaction. Oxaliplatin $134 \mathrm{mg}$ was administered following a 16-step desensitization protocol, as previously described [9] (fourth bag volume modified to $500 \mathrm{~mL}$ ) (Table), after premedication with intravenous dexamethasone $(16 \mathrm{mg})$, dexchlorpheniramine $(5 \mathrm{mg})$, ranitidine $(50 \mathrm{mg})$, granisetron (3 mg), acetaminophen (1 g), oral acetylsalicylic acid (300 mg), and montelukast (10 mg). Desensitization was completed at a final infusion rate of $160 \mathrm{~mL} / \mathrm{h}$, with no adverse events.

The second desensitization was performed 2 weeks later following the same protocol and premedication, although the final infusion rate was increased to $200 \mathrm{~mL} / \mathrm{h}$. Desensitization was completed. One hour later, the patient experienced nausea, profuse sweating and pallor, abdominal pain, and dizziness.
Her blood pressure dropped to $74 / 50 \mathrm{mmHg}$, and she had chills and fever at a body temperature of $38^{\circ} \mathrm{C}$. The oncologist suspected sepsis, and intensive fluid therapy was initiated, with a good response. Samples from peripheral blood, the Port-A-Cath reservoir, and urine were obtained for cultures. Meropenem was initiated immediately. The results of the blood test were as follows: white blood cell count, 16.7 (neutrophils $89 \%$ ); C-reactive protein, $1.4 \mathrm{mg} / \mathrm{mL}$; and procalcitonin, $0.64 \mu \mathrm{g} / \mathrm{L}$. Urinalysis and chest radiography were normal. The patient remained asymptomatic during the following days, and the results of culture were negative. She was discharged in 5 days with oral levofloxacin.

A third desensitization was performed 2 weeks later. An additional protocol step of $120 \mathrm{~mL} / \mathrm{h}$ was added with the fourth dilution bag. The final infusion rate was decreased to 160 $\mathrm{mL} / \mathrm{h}$. Intravenous dexketoprofen $50 \mathrm{mg}$ was administered after completion of the oxaliplatin infusion. Thirty minutes later, the patient developed nausea, dizziness, profuse sweating, and pallor, with a blood pressure of $55 / 35 \mathrm{mmHg}$ and heart rate of $35 \mathrm{bpm}$. Intramuscular epinephrine and intravenous saline, dexchlorpheniramine, and hydrocortisone were administered. The patient's condition improved after 30

Table. Oxaliplatin Desensitization Protocola

\begin{tabular}{|c|c|c|c|c|c|c|}
\hline & \multicolumn{6}{|c|}{ Total Dose, $134 \mathrm{mg}$} \\
\hline & & & Volume & Concent & & \\
\hline & & Solution 1 & $250 \mathrm{~mL}$ & $0.0005 \mathrm{n}$ & & \\
\hline & & Solution 2 & $250 \mathrm{~mL}$ & $0.005 \mathrm{~m}$ & & \\
\hline & & Solution 3 & $250 \mathrm{~mL}$ & $0.054 \mathrm{~m}$ & & \\
\hline & & Solution 4 & $500 \mathrm{~mL}$ & $0.265 \mathrm{~m}$ & & \\
\hline Step & Solution & Rate, $\mathrm{mL} / \mathrm{h}$ & Time, $\min$ & $\begin{array}{l}\text { Cumulative } \\
\text { Volume, } \mathrm{mL}\end{array}$ & Dose, $\mathrm{mg}$ & $\begin{array}{c}\text { Cumulative } \\
\text { Dose, mg }\end{array}$ \\
\hline 1 & 1 & 1 & 15 & 0.25 & 0.00013 & 0.00013 \\
\hline 2 & 1 & 2 & 15 & 0.75 & 0.00026 & 0.00026 \\
\hline 3 & 1 & 5 & 15 & 2 & 0.00065 & 0.001 \\
\hline 4 & 1 & 10 & 15 & 4.5 & 0.0013 & 0.002 \\
\hline 5 & 2 & 2 & 15 & 5 & 0.003 & 0.005 \\
\hline 6 & 2 & 5 & 15 & 6.25 & 0.007 & 0.012 \\
\hline 7 & 2 & 10 & 15 & 8.75 & 0.013 & 0.025 \\
\hline 8 & 2 & 20 & 15 & 13.75 & 0.027 & 0.052 \\
\hline 9 & 3 & 5 & 15 & 15 & 0.067 & 0.119 \\
\hline 10 & 3 & 10 & 15 & 17.5 & 0.134 & 0.253 \\
\hline 11 & 3 & 20 & 15 & 22.5 & 0.268 & 0.521 \\
\hline 12 & 3 & 40 & 15 & 32.5 & 0.536 & 1.057 \\
\hline 13 & 4 & 20 & 15 & 37.5 & 1.329 & 2.386 \\
\hline 14 & 4 & 40 & 15 & 47.5 & 2.659 & 5.045 \\
\hline 15 & 4 & 80 & 15 & 67.5 & 5.318 & 10.363 \\
\hline 16 & 4 & 160 & 174.375 & 532.5 & 123.636 & 134.000 \\
\hline
\end{tabular}

${ }^{a}$ Sixteen-step desensitization protocol for oxaliplatin. Four $1 / 10$ sequential dilutions were prepared. The volume for each dilution was $250 \mathrm{~mL}$, except for the fourth dilution, which was $500 \mathrm{~mL}$. The infusion rate was increased every 15 minutes, thus doubling the dose administered with each step. 
minutes, although hypotension remained largely unchanged $(70 / 30 \mathrm{mmHg})$. Nausea, dizziness, and abdominal pain resolved 1 hour later with saline and epinephrine. The patient remained in observation overnight, with no further adverse events.

Serum tryptase samples were not drawn after the anaphylactic episodes, although baseline serum tryptase $(3.21 \mu \mathrm{g} / \mathrm{L})$ and total $\operatorname{IgE}(57.3 \mathrm{kU} / \mathrm{L})$ were subsequently measured.

Given the recurrence of unexpected severe anaphylaxis after completion of the desensitization protocol and despite a 16-step protocol with full premedication and a favorable response of the tumor to FOFIRINOX-bevacizumab after proving refractory to all other therapies, it was decided to prescribe omalizumab as an adjuvant for desensitization. Omalizumab $300 \mathrm{mg}$ every 15 days was initiated 1 week before the following desensitization. The same protocol and premedication were followed, with good tolerance and no adverse reactions. Omalizumab was continued, and the patient has tolerated 13 further desensitizations to date. After 3 uneventful desensitizations, the patient developed fever $\left(39^{\circ} \mathrm{C}\right) 3$ hours after the oxaliplatin infusion, with no other symptoms or hypotension. The fever resolved with intravenous acetaminophen. Methylprednisolone $40 \mathrm{mg}$ and acetaminophen $1 \mathrm{~g}$ were prescribed after desensitization, and the patient did not experience fever during subsequent administrations.

Desensitization is standard of care for patients with drug allergy whenever they need the drug and alternative therapy is either unavailable or less effective [9]. Desensitization protocols are usually safe, and premedication helps to achieve tolerance with no or mild reactions in most patients [9]. However, a few patients with severe drug reactions cannot be managed despite adequately modified desensitization protocols and complete premedication.

Omalizumab has rarely been reported to achieve drug desensitization in patients with severe anaphylaxis and persistent reactions during attempted desensitization to oxaliplatin [4], carboplatin [5], and meropenem [6]. It has proven useful for achieving desensitization to acetylsalicylic acid and insulin that previously failed due to recurrent urticaria $[7,8]$.

In the present report, a patient with an initial immediate anaphylactic reaction to oxaliplatin presented unexpected anaphylaxis after completing a desensitization protocol with oxaliplatin. A positive intradermal test result with oxaliplatin suggests IgE-mediated anaphylaxis. Omalizumab helped to achieve desensitization to oxaliplatin.

Cahill et al [4] reported a case of anaphylaxis to oxaliplatin in which the patient developed severe back pain, hypoxemia, cough, chills, and hypotension during a 12-step desensitization protocol [4]. Omalizumab was followed by a 16-step desensitization protocol, leading to tolerance during 4 subsequent desensitizations. Skin tests with oxaliplatin were negative in this case. It remains uncertain whether a false-negative skin test result was involved or omalizumab was also effective in non-IgE-mediated drug reactions. More studies are needed to reinforce the indication of omalizumab as an adjuvant in drug desensitizations, although this option can be considered in high-risk patients.

\section{Funding}

The authors declare that no funding was received for the present study.

\section{Conflicts of Interest}

The authors declare that they have no conflicts of interest.

\section{References}

1. Beck LA, Marcotte GV, MacGlashan D, Togias A, Saini S. Omalizumab-induced reductions in mast cell Fcepsilon RI expression and function. J Allergy Clin Immunol. 2004;114:527-30.

2. Serrano-Candelas $E$, Martinez-Aranguren $R$, Valero A, Bartra J, Gastaminza G, Goikoetxea MJ, et al. Comparable actions of omalizumab on mast cells and basophils. Clin Exp Allergy. 2016;46:92-102.

3. Stokes JR, Casale TB. The use of anti-lgE therapy beyond allergic asthma. J Allergy Clin Immunol Pract. 2015;3:162-6.

4. Cahill KN, Harrison $P$, de Asis $M$, Castells $M$. Use of omalizumab to achieve successful desensitization after oxaliplatin anaphylaxis. J Allergy Clin Immunol. 2012;129:supplement,AB103.

5. Ojaimi S, Harnett PR, Fulcher DA. Successful carboplatin desensitization by using omalizumab and paradoxical diminution of total IgE levels. J Allergy Clin Immunol Pract. 2014;2:105-6.

6. Hennessey LR, Honicky R, Sudhanthar S, Dumpit AP. Successful intravenous "rush" drug desensitization to meropenem following omalizumab administration. J Allergy Clin Immunol. 2006;117:S225

7. Guillén $D$, Bobolea I, Calderon O, Fiandor A, Cabañas R, Heredia $R$, et al. Aspirin desensitization achieved after omalizumab treatment in a patient with aspirin-exacerbated urticaria and respiratory disease. J Investig Allergol Clin Immunol. 2015;25:133-62.

8. Matheu $V$, Franco $A$, Pérez $E$, Hernández $M$, Barrios $Y$. Omalizumab for drug allergy. J Allergy Clin Immunol. 2007;120:1471-2.

9. Castells M, Tennant NM, Sloane DE, Hsu Fl, Barrett NA, Hong DI, et al. Hypersensitivity reactions to chemotherapy: outcomes and safety of rapid desensitization in 413 cases. J Allergy Clin Immunol. 2008;122:574-80.

- Manuscript received July 2, 2018; accepted for publication September 19, 2018.

Pilar Tornero Molina

Servicio de Alergia

Hospital General Universitario Gregorio Marañón

Dr. Esquerdo, 46

28007 Madrid, Spain

E-mail: torneromolina@gmail.com 